\title{
Generalized Block-Diagonalization Schemes for MIMO Relay Broadcasting Systems
}

\author{
Xianan Wang, Xiaoxiang Wang, Wenrong Gong, and Zijia Huang
}

The Key Laboratory of Universal Wireless Communications, Ministry of Education, Beijing University of Posts and Telecommunications (BUPT), Beijing 100876, China

Correspondence should be addressed to Xianan Wang; wxn@bupt.edu.cn

Received 7 November 2014; Accepted 18 December 2014; Published 31 December 2014

Academic Editor: Dimitra Kaklamani

Copyright (C) 2014 Xianan Wang et al. This is an open access article distributed under the Creative Commons Attribution License, which permits unrestricted use, distribution, and reproduction in any medium, provided the original work is properly cited.

\begin{abstract}
We propose two generalized block-diagonalization (BD) schemes for multiple-input multiple-output (MIMO) relay broadcasting systems with no channel state information (CSI) at base station. We first introduce a generalized zero forcing (ZF) scheme that reduces the complexity of the traditional BD scheme. Then the optimal power loading matrix for the proposed scheme is analyzed and the closed-form solution is derived. Furthermore, an enhanced scheme is proposed by employing the minimum-mean-squarederror (MMSE) criterion. Simulation results show that the proposed generalized MMSE scheme outperforms the other schemes and the optimal power loading scheme improves the sum-rate performance efficiently.
\end{abstract}

\section{Introduction}

One major challenge faced by the wireless multimedia broadcasting systems is to provide high data rates for remote users located in the cell boundaries, which experience very low received signal-to-noise ratios (SNRs). An increasingly attractive and cost effective solution is the use of relay stations (RSs). Relays can be classified as full-duplex and half-duplex. Full-duplex relay is still under investigation due to its highly complex hardware implementation. For practical systems, half-duplex relay is more commonly used but suffers significant rate loss as a result of the two or more transmission phases needed to deliver a message. Multipleinput multiple-output (MIMO) technique is well known to provide significant improvement of the spectral efficiency and link reliability because of its multiplexing and diversity gains. So combining relay and MIMO techniques can utilize both of their advantages to increase the data rates of remote users.

From a general information theoretic perspective, the capacity bounds of MIMO relay channel with a single user have been analyzed in [1]. For practical implementation, [2] has investigated the optimal processing matrix at the relay in an amplify-and-forward (AF) relay MIMO system, also with a single user. When multiple antennas are deployed at base station (BS) and RS, multiple users can be scheduled at the same time for simultaneous transmissions. However, the optimal processing problem becomes more complex because of the multiuser interference (MUI). For MIMO relay broadcasting systems where each user is equipped with a single antenna, the authors in [3] proposed an implementable system architecture by exploiting nonlinear precoding at BS and linear processing at RS, while [4] proposed an iterative algorithm for jointly optimizing the precoding matrix at BS and RS to maximize the system capacity. Recently, the studies in [5] generalized the single-antenna user case to multiantenna, and a block-diagonalization (BD) based precoding scheme was applied to transform the system into multiple parallel single-user channels.

All the above research assumed that BS knows the channel state information (CSI) that is needed to perform the precoding. However, to inform BS of the CSI between RS and users would be rather challenging, especially for highvelocity users. The scenario that only RS has the CSI of the links from BS to RS and from RS to users was considered in [6] and a BD based linear processing scheme is employed at RS.

In this paper, we extend the work in [6] to a generalized form for MIMO relay broadcasting systems with no CSI at BS. The AF relay is considered due to its simplicity and 


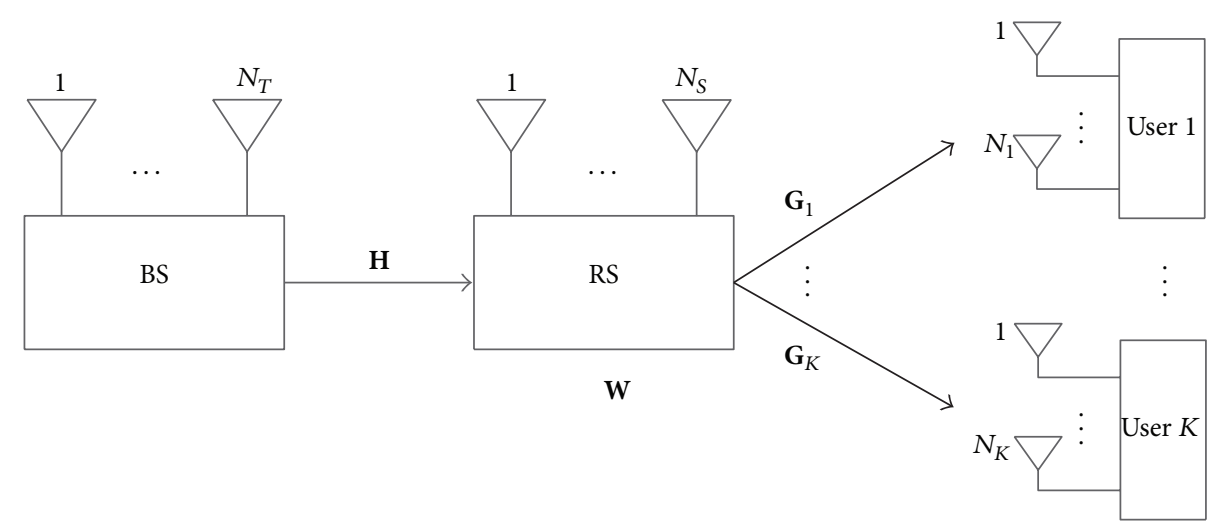

FIGURE 1: System model of the MIMO relay broadcasting system.

practicality. We first introduce a generalized zero forcing (ZF) scheme to reduce the complexity of the traditional BD based scheme. Then the optimal power loading matrix for the proposed scheme is analyzed and the closed-form solution is derived. Furthermore, by employing the minimum-meansquared-error (MMSE) criterion, an enhanced scheme is proposed to balance the interference and noise. Simulation results show that the proposed generalized MMSE scheme is superior to the other schemes and the optimal power loading scheme improves the sum-rate performance efficiently.

This paper is organized as follows. The system model and problem formulation are given in Section 2. In Section 3, a brief review of the traditional $\mathrm{BD}$ based scheme is presented. The generalized BD schemes are developed in Section 4. Simulation results and conclusions are displayed in Section 5 and Section 6, respectively.

The following notations are used throughout the paper. Boldface capitals and lowercases denote matrices and vectors. $\mathbf{X}^{T}, \mathbf{X}^{H}, \mathbf{X}^{-1}$, and $\mathbf{X}^{+}$denote the transpose, conjugate transpose, inverse, and pseudoinverse of $\mathbf{X}$, respectively. $\mathbf{I}$ represents the identity matrix.

\section{System Model and Problem Formulation}

We consider a MIMO relay broadcasting system as shown in Figure 1. In this system, the BS with $N_{T}$ antennas transmits independent data streams to $K$ remote users simultaneously and an RS with $N_{S}$ antennas helps the communication. The $k$ th user is equipped with $N_{k}$ antennas and the total number of receive antennas is $N_{R}=\sum_{k=1}^{K} N_{k}$. In this paper, we assume that $N_{T}=N_{R}$ for simplicity. For the case $N_{T} \neq$ $N_{R}$, a user scheduling algorithm is needed and the multiuser diversity gain can be exploited. The system configuration can be described as $N_{T} \times N_{S} \times\left\{N_{1}, \ldots, N_{K}\right\}$. We ignore the direct link between $\mathrm{BS}$ and each user due to very severe large-scale path loss.

The data transmitted from BS to the $k$ th user is assumed to be an $N_{k}$-dimensional symbol vector $\mathbf{x}_{k}$, which is normalized as $E\left[\mathbf{x}_{k} \mathbf{x}_{k}^{H}\right]=\mathbf{I}$. Therefore, the total transmitted data vector at
BS can be expressed as $\mathbf{x}=\left[\mathbf{x}_{1}^{T}, \ldots, \mathbf{x}_{K}^{T}\right]^{T}$. The received data vector at $\mathrm{RS}$ is given by

$$
\mathbf{y}_{r}=\sqrt{\frac{P_{T}}{N_{T}}} \mathbf{H x}+\mathbf{n}_{S},
$$

where $P_{T}$ is the transmit power at BS and $\mathbf{H} \in \mathbb{C}^{N_{S} \times N_{T}}$ is the backward channel matrix. $\mathbf{n}_{S}$ is the RS's complex Gaussian noise vector with independent and identically distributed (i.i.d.) elements of zero mean and variance $\sigma_{S}^{2}$.

After a linear processing, the transmitted data vector at $\mathrm{RS}$ is expressed as

$$
\mathbf{x}_{r}=\mathbf{W y}_{r}=\sqrt{\frac{P_{T}}{N_{T}}} \mathbf{W H x}+\mathbf{W n}_{S},
$$

where $\mathbf{W} \in \mathbb{C}^{N_{S} \times N_{S}}$ is the linear processing matrix at RS. The transmit power constraint at RS can be written as

$$
\operatorname{tr}\left(\frac{P_{T}}{N_{T}} \mathbf{W} \mathbf{H} \mathbf{H}^{H} \mathbf{W}^{H}+\sigma_{S}^{2} \mathbf{W} \mathbf{W}^{H}\right) \leq P_{S} .
$$

Finally, the received data vector at the $k$ th user is represented as

$$
\mathbf{x}_{k}=\mathbf{G}_{k} \mathbf{x}_{r}+\mathbf{n}_{R}=\sqrt{\frac{P_{T}}{N_{T}}} \mathbf{G}_{k} \mathbf{W H x}+\mathbf{G}_{k} \mathbf{W} \mathbf{n}_{S}+\mathbf{n}_{R},
$$

where $\mathbf{G}_{k} \in \mathbb{C}^{N_{k} \times N_{S}}$ is the forward channel matrix from RS to the $k$ th user and $\mathbf{n}_{R}$ is the user's complex Gaussian noise vector with i.i.d. elements of zero mean and variance $\sigma_{R}^{2}$. Equation (4) can be rewritten as

$$
\begin{aligned}
\mathbf{x}_{k}= & \sqrt{\frac{P_{T}}{N_{T}}} \mathbf{G}_{k} \mathbf{W H}_{k} \mathbf{x}_{k}+\sqrt{\frac{P_{T}}{N_{T}}} \mathbf{G}_{k} \mathbf{W} \sum_{j=1, j \neq k}^{K} \mathbf{H}_{j} \mathbf{x}_{j} \\
& +\mathbf{G}_{k} \mathbf{W n}_{S}+\mathbf{n}_{R},
\end{aligned}
$$

where $\mathbf{H}_{k} \in \mathbb{C}^{N_{S} \times N_{k}}$ represents the channel matrix from the specific $N_{k}$ transmit antennas at BS which are dedicated to the $k$ th user to the $N_{S}$ receive antennas at RS. 
As we can see from (5), the first term of the right-hand side indicates the desired data of the $k$ th user and the others include the MUI and noise. To fully eliminate the MUI in (5), W should satisfy

$$
\mathbf{G}_{k} \mathbf{W H}_{j} \begin{cases}\neq 0, & \text { for } j=k \\ =0, & \text { otherwise }\end{cases}
$$

or

$$
\mathbf{G W H}=\left[\begin{array}{ccc}
\mathbf{G}_{1} \mathbf{W H}_{1} & 0 & 0 \\
0 & \cdots & 0 \\
0 & 0 & \mathbf{G}_{K} \mathbf{W H}_{K}
\end{array}\right]
$$

where $\mathbf{G}=\left[\mathbf{G}_{1}^{T} \cdots \mathbf{G}_{K}^{T}\right]^{T}$ is the combining forward channel matrix. We define $\mathbf{W}$ as

$$
\mathbf{W}=\mathbf{W}_{G} \mathbf{W}_{H} \text {. }
$$

$\mathbf{W}_{G}$ and $\mathbf{W}_{H}$ are the linear processing matrices for the forward channel and the backward channel, respectively. It is obvious that (7) is satisfied when we set $\mathbf{W}_{G}=\mathbf{G}^{+}$ and $\mathbf{W}_{H}=\mathbf{H}^{+}$. However, this scheme simply treats each antenna as a single receiver, thus sacrificing some benefit of the multiantenna at each user.

\section{Review of the BD Based Scheme}

The authors in [6] proposed a BD based scheme, where $\mathbf{W}_{G}$ and $\mathbf{W}_{H}$ are designed to place each user's data stream at the null space of the other users' channels.

To this end, we exclude the $k$ th user's channel matrix from $\mathrm{G}$ and define

$$
\overline{\mathbf{G}}_{k}=\left[\begin{array}{llllll}
\mathbf{G}_{1}^{T} & \cdots & \mathbf{G}_{k-1}^{T} & \mathbf{G}_{k+1}^{T} & \cdots & \mathbf{G}_{K}^{T}
\end{array}\right]^{T} \in \mathbb{C}^{\bar{N}_{G k} \times N_{s}},
$$

where $\bar{N}_{G k}=N_{R}-N_{k}$. Assuming that $N_{S} \geq N_{R}$, we have rank $\left(\overline{\mathbf{G}}_{k}\right)=\bar{N}_{G k}$ in a rich scattering environment. The singular value decomposition (SVD) of $\overline{\mathbf{G}}_{k}$ is

$$
\overline{\mathbf{G}}_{k}=\mathbf{U}_{G k} \Sigma_{G k}\left[\begin{array}{ll}
\mathbf{V}_{G k, 1} & \mathbf{V}_{G k, 0}
\end{array}\right]^{H},
$$

where $\mathbf{U}_{G k} \in \mathbb{C}^{\bar{N}_{G k} \times \bar{N}_{G k}}$ is a unitary matrix and the diagonal matrix $\Sigma_{G k} \in \mathbb{C}^{\bar{N}_{G k} \times N_{S}}$ contains the singular values of $\overline{\mathbf{G}}_{k}$. $\mathbf{V}_{G k, 1} \in \mathbb{C}^{N_{S} \times \bar{N}_{G k}}$ consists of the first $\bar{N}_{G k}$ nonzero singular vectors and $\mathbf{V}_{G k, 0} \in \mathbb{C}^{N_{S} \times\left(N_{S}-\bar{N}_{G k}\right)}$ holds the last $N_{S}-\bar{N}_{G k}$ zero singular vectors. Thus, $\mathbf{V}_{G k, 0}$ forms an orthonormal basis for the null space of $\overline{\mathbf{G}}_{k}$ and $\mathbf{W}_{G}$ can be expressed as

$$
\mathbf{W}_{G}=\left[\begin{array}{lll}
\mathbf{V}_{G 1,0} & \cdots & \mathbf{V}_{G K, 0}
\end{array}\right] .
$$

$\mathbf{W}_{H}$ can be calculated in a similar way. We define

$$
\overline{\mathbf{H}}_{k}=\left[\begin{array}{llllll}
\mathbf{H}_{1} & \cdots & \mathbf{H}_{k-1} & \mathbf{H}_{k+1} & \cdots & \mathbf{H}_{K}
\end{array}\right] \in \mathbb{C}^{N_{S} \times \bar{N}_{H k}},
$$

where $\bar{N}_{H k}=N_{T}-N_{k}$. Assuming that $N_{S} \geq N_{T}$, we have $\operatorname{rank}\left(\overline{\mathbf{H}}_{k}\right)=\bar{N}_{H k}$ in a rich scattering environment. The SVD of $\overline{\mathbf{H}}_{k}$ is

$$
\overline{\mathbf{H}}_{k}=\left[\begin{array}{ll}
\mathbf{U}_{H k, 1} & \mathbf{U}_{H k, 0}
\end{array}\right] \boldsymbol{\Sigma}_{H k} \mathbf{V}_{H k}^{H},
$$

where $\mathbf{U}_{H k, 0}^{H} \in \mathbb{C}^{\left(N_{S}-\bar{N}_{H k}\right) \times N_{S}}$ forms an orthonormal basis for the null space of $\overline{\mathbf{H}}_{k}$ and $\mathbf{W}_{H}$ can be expressed as

$$
\mathbf{W}_{H}=\left[\begin{array}{lll}
\mathbf{U}_{H 1,0} & \cdots & \mathbf{U}_{H K, 0}
\end{array}\right]^{H} .
$$

After the determination of $\mathbf{W}_{G}$ and $\mathbf{W}_{H}$, the MUI at each user is fully eliminated. Since this BD based scheme uses SVD to calculate the linear processing matrix, we term it as the $\mathrm{BD}$ SVD scheme.

\section{Generalized BD Schemes}

In this section, we introduce the generalized BD schemes to reduce the complexity and improve the performance of MIMO relay broadcasting systems.

4.1. Generalized ZF Scheme. Although the BD-SVD scheme can fully eliminate the MUI and benefit from the multiantenna gain, the SVD operations bring along considerable computational complexity which makes it difficult to implement in practice. In order to reduce the complexity, we propose a $\mathrm{BD}$ based generalized $\mathrm{ZF}$ scheme (BD-GZF) as follows.

We first define the pseudoinverse of the forward channel matrix $\mathbf{G}$ as

$$
\mathbf{G}^{+}=\mathbf{G}^{H}\left(\mathbf{G G}^{H}\right)^{-1}=\left[\begin{array}{lll}
\mathbf{G}_{1}^{+} & \cdots & \mathbf{G}_{K}^{+}
\end{array}\right] .
$$

By performing $\mathrm{QR}$ decomposition (QRD) on $\mathbf{G}_{k}^{+}$, we get

$$
\mathbf{G}_{k}^{+}=\mathbf{Q}_{G k} \mathbf{R}_{G k}, \quad k=1, \ldots, K,
$$

where $\mathbf{Q}_{G k} \in \mathbb{C}^{N_{S} \times N_{k}}$ forms an orthonormal basis for the column space of $\mathbf{G}_{k}^{+}$and $\mathbf{R}_{G k} \in \mathbb{C}^{N_{k} \times N_{k}}$ is an upper triangular matrix [7]. It is observed that $\mathbf{G}_{j} \mathbf{G}_{k}^{+}=\mathbf{G}_{j} \mathbf{Q}_{G k} \mathbf{R}_{G k}=\mathbf{0}$, for $j \neq k$. Since $\mathbf{R}_{G k}$ is nonsingular, it follows $\mathbf{G}_{j} \mathbf{Q}_{G k}=\mathbf{0}$. Therefore, $\mathbf{Q}_{G k}$ forms an orthonormal basis for the null space of $\overline{\mathbf{G}}_{k}$, just like $\mathbf{V}_{G k, 0}$ in the BD-SVD scheme. Thus $\mathbf{W}_{G}$ can be obtained as

$$
\mathbf{W}_{G}=\left[\begin{array}{lll}
\mathbf{Q}_{G 1} & \cdots & \mathbf{Q}_{G K}
\end{array}\right] .
$$

$\mathbf{W}_{H}$ can be calculated in a similar way. We define

$$
\mathbf{H}^{+}=\left(\mathbf{H}^{H} \mathbf{H}\right)^{-1} \mathbf{H}^{H}=\left[\begin{array}{lll}
\mathbf{H}_{1}^{+} & \cdots & \mathbf{H}_{K}^{+}
\end{array}\right]^{H} .
$$

By performing QRD operation on $\mathbf{H}_{k}^{+}$, we get

$$
\mathbf{H}_{k}^{+}=\mathbf{Q}_{H k} \mathbf{R}_{H k}, \quad k=1, \ldots, K,
$$

where $\mathbf{Q}_{H k} \in \mathbb{C}^{N_{S} \times N_{k}}$ forms an orthonormal basis for the null space of $\overline{\mathbf{H}}_{k}$ and $\mathbf{W}_{H}$ can be obtained as

$$
\mathbf{W}_{H}=\left[\begin{array}{lll}
\mathbf{Q}_{H 1} & \cdots & \mathbf{Q}_{H K}
\end{array}\right]^{H} .
$$

4.2. Complexity Analysis. We use the number of floating point operations (FLOPs) to measure the computational 
TABLE 1: Complexity of BD-SVD.

\begin{tabular}{lcc}
\hline Steps & Operations & FLOPs \\
\hline 1 & $\begin{array}{c}\text { SVD for } \\
\text { calculating } \mathbf{W}_{G}\end{array}$ & $32 \sum_{k=1}^{K}\left(N_{S} \bar{N}_{G k}^{2}+2 \bar{N}_{G k}^{3}\right)$ \\
\hline 2 & $\begin{array}{c}\text { SVD for } \\
\text { calculating } \mathbf{W}_{H}\end{array}$ & $32 \sum_{k=1}^{K}\left(N_{S} \bar{N}_{H k}^{2}+2 \bar{N}_{H k}^{3}\right)$ \\
\hline
\end{tabular}

TABLE 2: Complexity of BD-GZF.

\begin{tabular}{lcc}
\hline Steps & Operations & FLOPs \\
\hline 1 & Inversion of $\mathbf{G}$ & $4 / 3 N_{R}^{3}+16 N_{R}^{2} N_{S}-2 N_{R}^{2}-2 N_{R} N_{S}$ \\
\hline 2 & QRD of $\mathbf{W}_{G}$ & $16 \sum_{k=1}^{K}\left(N_{S}^{2} N_{k}-N_{S} N_{k}^{2}+1 / 3 N_{k}^{3}\right)$ \\
\hline 3 & Inversion of $\mathbf{H}$ & $4 / 3 N_{T}^{3}+16 N_{T}^{2} N_{S}-2 N_{T}^{2}-2 N_{T} N_{S}$ \\
\hline 4 & QRD of $\mathbf{W}_{H}$ & $16 \sum_{k=1}^{K}\left(N_{S}^{2} N_{k}-N_{S} N_{k}^{2}+1 / 3 N_{k}^{3}\right)$ \\
\hline
\end{tabular}

complexity. According to [8], the numbers of FLOPs required for different matrix operations are summarized as follows:

(i) multiplication of $m \times n$ and $n \times p$ complex matrices: $8 m n p-2 m p$

(ii) SVD of an $m \times n(m \leq n)$ complex matrix where only $\boldsymbol{\Sigma}$ and $\mathbf{V}$ are obtained: $32\left(n m^{2}+2 m^{3}\right)$;

(iii) inversion of an $m \times m$ real matrix: $4 m^{3} / 3$;

(iv) QRD of an $m \times n(m \leq n)$ complex matrix: $16\left(n^{2} m-\right.$ $\left.n m^{2}+1 / 3 m^{3}\right)$.

The required numbers of FLOPs for the BD-SVD and BDGZF schemes are illustrated in Tables 1 and 2, respectively.

In order to make the complexity comparison more comprehensive and intuitive, we plot the numbers of FLOPs required for the two schemes in Figure 2 as a function of user number. We assume that each user is equipped with $N_{k}=2$ antennas and $N_{T}=N_{S}=N_{k} \times K$.

It can be seen from Figure 2 that the proposed BD-GZF scheme demands much lower computational complexity than the BD-SVD scheme. One reason is that the QRD operation is much simpler than the SVD operation in the case of same matrix dimension. A more important reason is that the SVD operations in the BD-SVD scheme are implemented $K$ times on matrices with dimensions $\bar{N}_{G k} \times N_{S}$ and $N_{S} \times \bar{N}_{H k}$, while the QRD operations in the BD-GZF scheme are implemented $K$ times on matrices with dimensions $N_{S} \times N_{k}$ and $N_{S} \times N_{k}$, which are much lower than the former. It is worth noting that, with the increase of the system dimension, the complexity reduction by the proposed $\mathrm{BD}-\mathrm{GZF}$ scheme becomes more considerable.

4.3. Optimal Power Loading Scheme. To maximize the sumrate, we define the power loading matrix $\mathbf{P}$ as

$$
\mathbf{P}=\left[\begin{array}{ccc}
\mathbf{P}_{1} & 0 & 0 \\
0 & \cdots & 0 \\
0 & 0 & \mathbf{P}_{K}
\end{array}\right]
$$

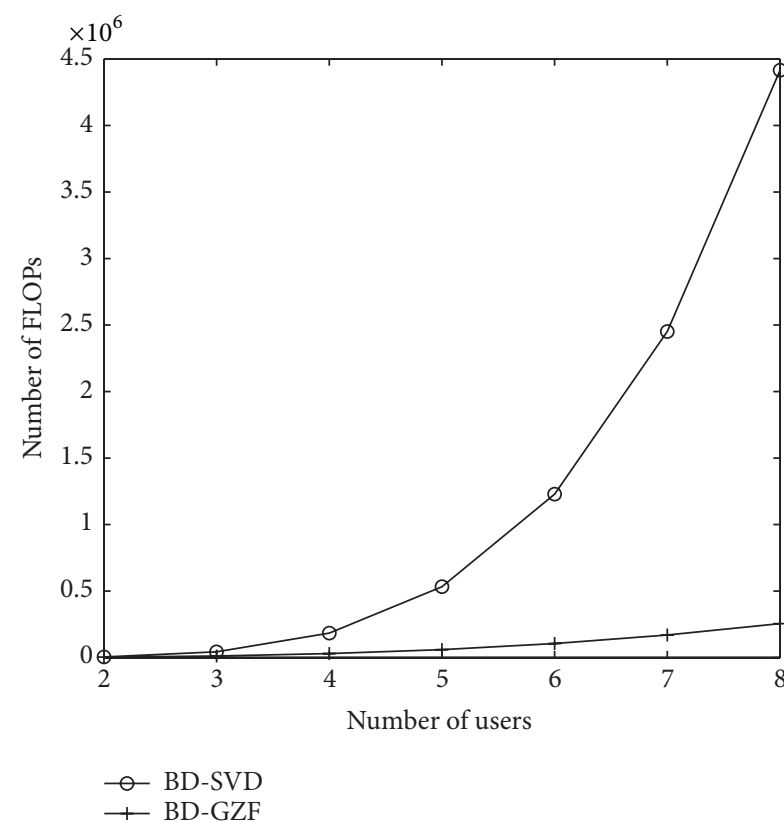

FIgURE 2: Complexity comparison with $N_{k}=2$ and $N_{T}=N_{S}=$ $N_{k} \times K$.

where $\mathbf{P}_{k} \in \mathbb{C}^{N_{k} \times N_{k}}$ is the power loading matrix for the $k$ th user. With the power loading matrix being introduced, (8) can be rewritten as

$$
\mathbf{W}=\mathbf{W}_{G} \mathbf{P} \mathbf{W}_{H} \text {. }
$$

By substituting (17), (20), (21), and (22) into (5), we get

$$
\mathbf{y}_{k}=\sqrt{\frac{P_{T}}{N_{T}}} \widetilde{\mathbf{G}}_{k} \mathbf{P}_{k} \widetilde{\mathbf{H}}_{k} \mathbf{x}_{k}+\widetilde{\mathbf{G}}_{k} \mathbf{P}_{k} \mathbf{Q}_{H k}^{H} \mathbf{n}_{S}+\mathbf{n}_{R},
$$

where $\widetilde{\mathbf{G}}_{k}=\mathbf{G}_{k} \mathbf{Q}_{G k}$ and $\widetilde{\mathbf{H}}_{k}=\mathbf{Q}_{H k}^{H} \mathbf{H}_{k}$ denote the effective channel matrix for the forward and backward channels, respectively. As we can see, after the linear processing, the MIMO relay broadcasting system becomes $K$ parallel MIMO relay systems. Motivated by the optimal design for the singleuser MIMO relay system [2], we perform SVD operations on $\widetilde{\mathbf{G}}_{k}$ and $\widetilde{\mathbf{H}}_{k}$ as $\widetilde{\mathbf{G}}_{k}=\widetilde{\mathbf{U}}_{G K} \widetilde{\Sigma}_{G K} \widetilde{\mathbf{V}}_{G K}^{H}$ and $\widetilde{\mathbf{H}}_{k}=\widetilde{\mathbf{U}}_{H K} \widetilde{\Sigma}_{H K} \widetilde{\mathbf{V}}_{H K}^{H}$. Then the optimal $\mathbf{P}_{k}$ can be expressed as

$$
\mathbf{P}_{k}=\widetilde{\mathbf{V}}_{G k} \boldsymbol{\Lambda}_{k} \widetilde{\mathbf{U}}_{H k}^{H}
$$

where $\Lambda_{k}=\operatorname{diag}\left(\sqrt{\Lambda_{k, 1}}, \ldots, \sqrt{\Lambda_{k, N_{k}}}\right)$ is for allocating the power. Thus (23) can be rewritten as

$$
\begin{aligned}
\mathbf{y}_{k}= & \sqrt{\frac{P_{T}}{N_{T}}} \widetilde{\mathbf{U}}_{G K} \widetilde{\boldsymbol{\Sigma}}_{G K} \boldsymbol{\Lambda}_{k} \widetilde{\boldsymbol{\Sigma}}_{H K} \widetilde{\mathbf{V}}_{H K}^{H} \mathbf{x}_{k} \\
& +\widetilde{\mathbf{U}}_{G K} \widetilde{\boldsymbol{\Sigma}}_{G K} \boldsymbol{\Lambda}_{k} \widetilde{\mathbf{U}}_{H k}^{H} \mathbf{Q}_{H k}^{H} \mathbf{n}_{S}+\mathbf{n}_{R} .
\end{aligned}
$$


The sum-rate at the $k$ th user is derived as

$$
\begin{aligned}
R_{k} & =\frac{1}{2} \log _{2} \operatorname{det}\left(\mathbf{I}+\frac{\left(P_{T} / N_{T}\right) \widetilde{\Sigma}_{H K}^{2} \Lambda_{k}^{2} \widetilde{\Sigma}_{G K}^{2}}{\sigma_{S}^{2} \Lambda_{k}^{2} \widetilde{\Sigma}_{G K}^{2}+\sigma_{R}^{2}}\right) \\
& =\frac{1}{2} \sum_{j=1}^{N_{k}} \log _{2}\left(1+\frac{\left(P_{T} / N_{T}\right) \widetilde{\Sigma}_{H k, j} \Lambda_{k, j} \widetilde{\Sigma}_{G k, j}}{\sigma_{S}^{2} \Lambda_{k, j} \widetilde{\Sigma}_{G k, j}+\sigma_{R}^{2}}\right),
\end{aligned}
$$

where the factor of $1 / 2$ comes from the loss of the half-duplex transmission. $\widetilde{\Sigma}_{H k, j}$ and $\widetilde{\Sigma}_{G k, j}$ are the $j$ th elements of the diagonal matrices $\widetilde{\Sigma}_{H K}^{2}$ and $\widetilde{\Sigma}_{G K}^{2}$, respectively.

The transmit power constraint at RS can be written as

$$
\begin{aligned}
\sum_{k=1}^{K} \operatorname{tr}\left(\frac{P_{T}}{N_{T}} \Lambda_{k}^{2} \widetilde{\Sigma}_{H k}^{2}+\sigma_{S}^{2} \Lambda_{k}^{2}\right) \\
=\sum_{k=1}^{K} \sum_{j=1}^{N_{k}} \Lambda_{k, j}\left(\frac{P_{T}}{N_{T}} \widetilde{\Sigma}_{H k, j}+\sigma_{S}^{2}\right) \leq P_{S} .
\end{aligned}
$$

Therefore, the maximizing problem of the system sumrate $R=\sum_{k=1}^{K} R_{k}$ is formulated as

$$
\begin{array}{ll}
\underset{\Lambda_{k, j}}{\operatorname{maxmize}} & R \\
\text { subject to } & \Lambda_{k, j} \geq 0, \quad \forall k, j \\
& \sum_{k=1}^{K} \sum_{j=1}^{N_{k}} \Lambda_{k, j}\left(\frac{P_{T}}{N_{T}} \widetilde{\Sigma}_{H k, j}+\sigma_{S}^{2}\right) \leq P_{S} .
\end{array}
$$

Problem (28) is a standard convex optimization problem and can be solved with the Lagrange multiplier method. The closed-form solution is given as

$\Lambda_{k, j}$

$$
=\frac{\sigma_{R}^{2}\left(\sqrt{\left(\rho \widetilde{\Sigma}_{H k, j}\right)^{2}+4 \rho \mu \widetilde{\Sigma}_{H k, j} \widetilde{\Sigma}_{G k, j} / \ln 2 \sigma_{R}^{2}}-\rho \widetilde{\Sigma}_{H k, j}-2\right)^{+}}{2 \sigma_{S}^{2} \widetilde{\Sigma}_{G k, j}\left(1+\rho \widetilde{\Sigma}_{H k, j}\right)},
$$

where $\rho=P_{T} /\left(N_{T} \sigma_{S}^{2}\right)$ and $(x)^{+}=\max (0, x) \cdot \mu$ is a unique root of $\sum_{k=1}^{K} \sum_{j=1}^{N_{k}} \Lambda_{k, j}\left(P_{T} \widetilde{\Sigma}_{H k, j} / N_{T}+\sigma_{S}^{2}\right)-P_{S}=0$, which can be solved with a numerical root-finding algorithm, such as the bisection method.

4.4. Enhanced MMSE Scheme. It is well known that the ZF criterion eliminates the MUI without appropriate consideration of the noise and therefore some performance loss will occur. To take the noise term into account and improve the performance, an enhanced scheme termed the BD based generalized MMSE scheme (BD-GMMSE) is proposed as follows.

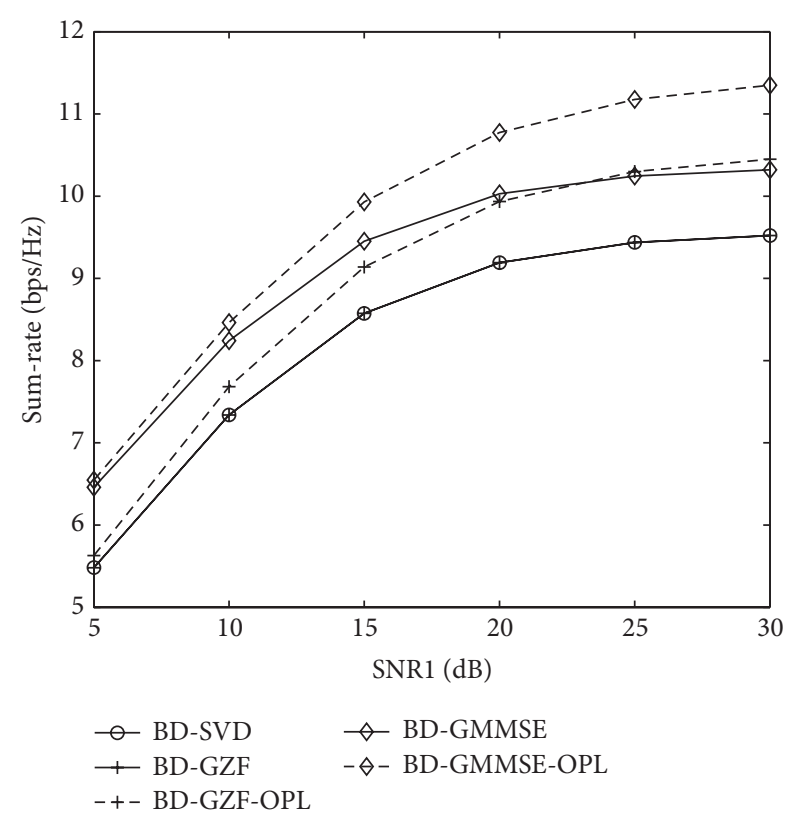

FIGURE 3: Sum-rate as a function of SNR1 with SNR2 fixed at $10 \mathrm{~dB}$.

By applying the MMSE criterion, we replace the pseudoinverse in (15) and (18) with MMSE channel inversion as [9]

$$
\begin{aligned}
& \mathbf{G}_{\text {mmse }}^{+}=\mathbf{G}^{H}\left(\mathbf{G G}^{H}+\alpha \mathbf{I}\right)^{-1}, \\
& \mathbf{H}_{\text {mmse }}^{+}=\left(\mathbf{H}^{H} \mathbf{H}+\beta \mathbf{I}\right)^{-1} \mathbf{H}^{H},
\end{aligned}
$$

where $\alpha=N_{S} \sigma_{R}^{2} / P_{S}$ and $\beta=N_{T} \sigma_{S}^{2} / P_{T}$. Then $\mathbf{W}_{G}$ and $\mathbf{W}_{H}$ can be obtained in the same manner.

Note that the BD-GZF scheme relies on the pseudoinverse of $\mathbf{G}$ and $\mathbf{H}$, thus having the dimension constraints; that is, $N_{S} \leq N_{T}$ and $N_{R} \leq N_{S}$. However, the BD-GMMSE scheme can be employed when there are more receive antennas than transmit antennas, similar to the MMSE channel inversion.

Unlike the BD-GZF scheme, the optimal power loading matrix for the BD-GMMSE scheme is not easy to identify since the residual MUI varies according to the power loading. However, the above optimal power loading scheme can still be applied in BD-GMMSE and improve the sum-rate performance efficiently as will be shown later.

\section{Simulation Results}

In this section, we present simulation results to show the sum-rate performance of the proposed schemes. A system under the configuration of $6 \times 6 \times\{2,2,2\}$ is considered. All channels are assumed to be quasistatic flat faded and the elements are complex Gaussian variables with zero mean and unit variance. The average received SNRs per antenna at RS and at users are denoted by SNR1 and SNR2, respectively. OPL in the legend denotes the implementation of the proposed optimal power loading scheme and the bisection method is used to calculate $\mu$. We average the sum-rate over 10000 random channel realizations. 


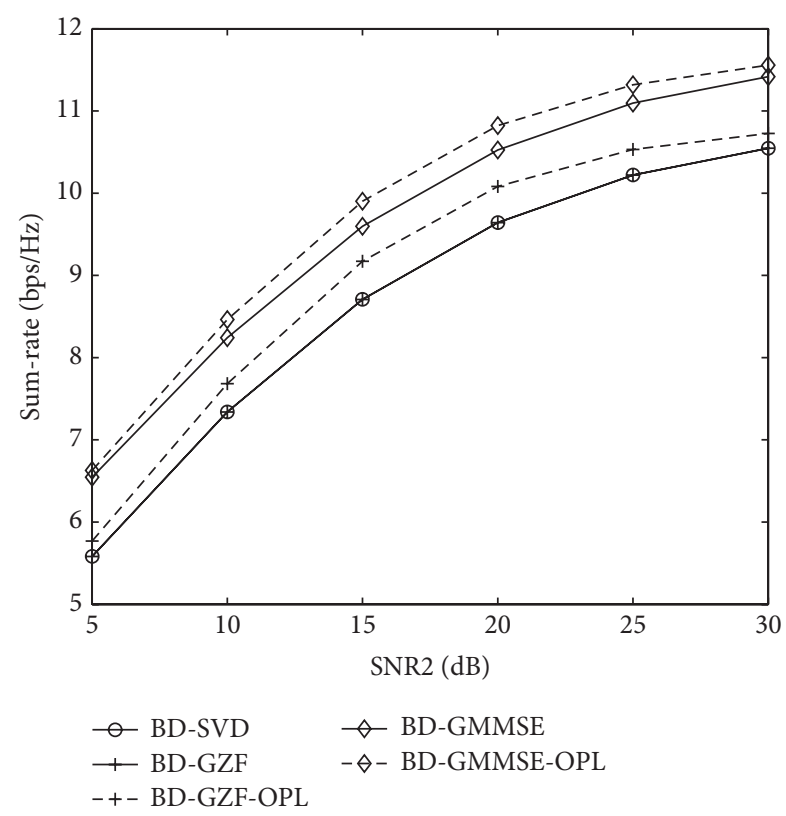

FIGURE 4: Sum-rate as a function of SNR2 with SNR1 fixed at $10 \mathrm{~dB}$.

Figure 3 shows the sum-rate performance of different schemes as a function of SNR1 with SNR2 fixed at $10 \mathrm{~dB}$, and vice versa in Figure 4 . It can be seen that the proposed BD-GZF scheme represents identical performance as the traditional BD-SVD scheme, while offering much lower computational complexity as analyzed in Section 4 . The enhanced BD-GMMSE scheme outperforms ZF based schemes by balancing the interference and noise. The proposed optimal power loading scheme improves the sum-rate performance efficiently for both BD-GZF and BD-GMMSE schemes.

\section{Conclusion}

In this paper, two generalized BD schemes for MIMO relay broadcasting systems are proposed. We first extend the traditional BD based scheme to a generalized BD-GZF scheme, which reduces the computational complexity significantly. Then an optimal power loading scheme for the proposed scheme is developed to improve the sum-rate performance. By employing the MMSE criterion, an enhanced scheme is proposed to further improve the performance. Simulation results show that the proposed BD-GMMSE scheme is superior to the other schemes and the optimal power loading scheme improves the sum-rate performance efficiently.

\section{Conflict of Interests}

The authors declare that there is no conflict of interests regarding the publication of this paper.

\section{Acknowledgments}

This work is supported by the 863 Project (no. 2014AA01A701) and the National Natural Science Foundation of China (no. 61072052).

\section{References}

[1] B. Wang, J. Zhang, and A. Host-Madsen, "On the capacity of MIMO relay channels," IEEE Transactions on Information Theory, vol. 51, no. 1, pp. 29-43, 2005.

[2] X. Tang and Y. Hua, "Optimal design of non-regenerative MIMO wireless relays," IEEE Transactions on Wireless Communications, vol. 6, no. 4, pp. 1398-1406, 2007.

[3] C.-B. Chae, T. Tang, J. Heath, and S. Cho, "MIMO relaying with linear processing for multiuser transmission in fixed relay networks," IEEE Transactions on Signal Processing, vol. 56, no. 2, pp. 727-738, 2008.

[4] W. Xu, X. Dong, and W.-S. Lu, "Joint precoding optimization for multiuser multi-antenna relaying downlinks using quadratic programming," IEEE Transactions on Communications, vol. 59, no. 5, pp. 1228-1235, 2011.

[5] L. Liang, W. Xu, and X. Dong, "Limited feedback-based multiantenna relay broadcast channels with block diagonalization," IEEE Transactions on Wireless Communications, vol. 12, no. 8, pp. 4092-4101, 2013.

[6] W. Liu, C. Li, J.-D. Li, and L. Hanzo, "Block diagonalisationbased multiuser multiple input multiple output-aided downlink relaying," IET Communications, vol. 6, no. 15, pp. 2371-2377, 2012.

[7] H. Sung, S.-R. Lee, and I. Lee, "Generalized channel inversion methods for multiuser MIMO systems," IEEE Transactions on Communications, vol. 57, no. 11, pp. 3489-3499, 2009.

[8] G. Golub and C. V. Loan, Matrix Computations, The Johns Hopkins University Press, 1996.

[9] C. B. Peel, B. M. Hochwald, and A. L. Swindlehurst, "A vector-perturbation technique for near-capacity multiantenna multiuser communication-part I: channel inversion and regularization," IEEE Transactions on Communications, vol. 53, no. 1, pp. 195-202, 2005. 

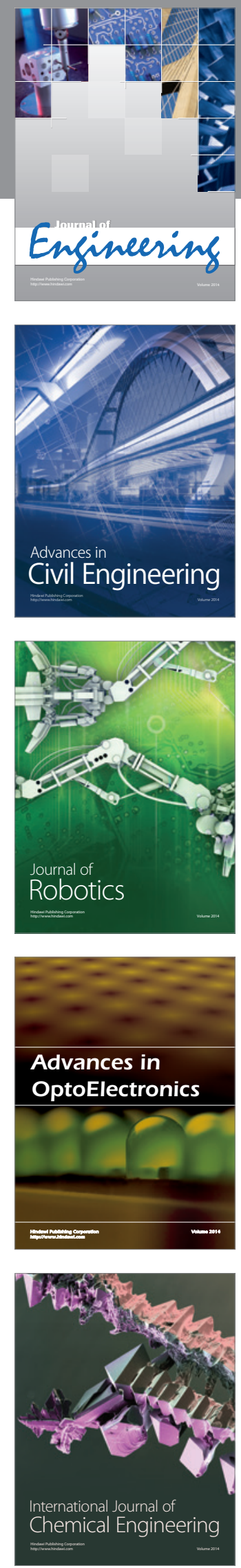

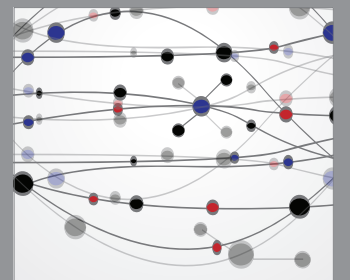

The Scientific World Journal
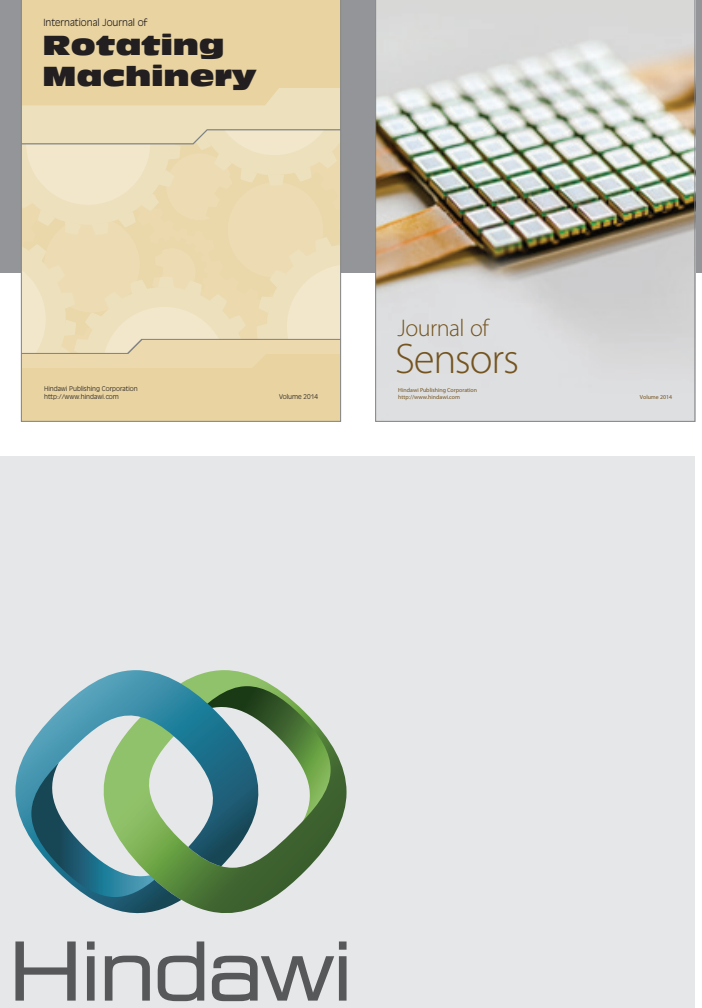

Submit your manuscripts at http://www.hindawi.com
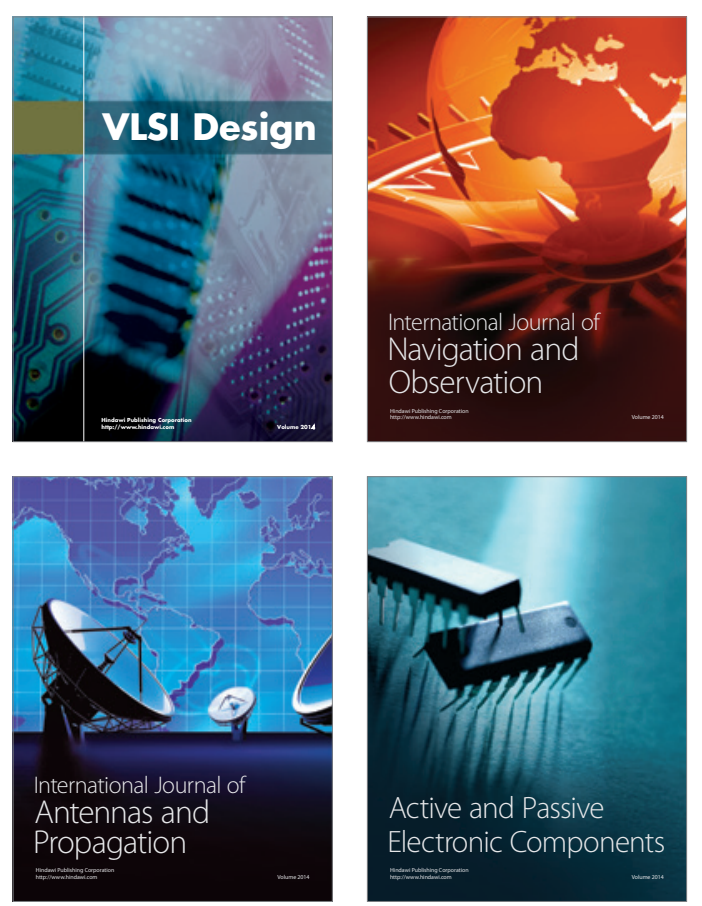
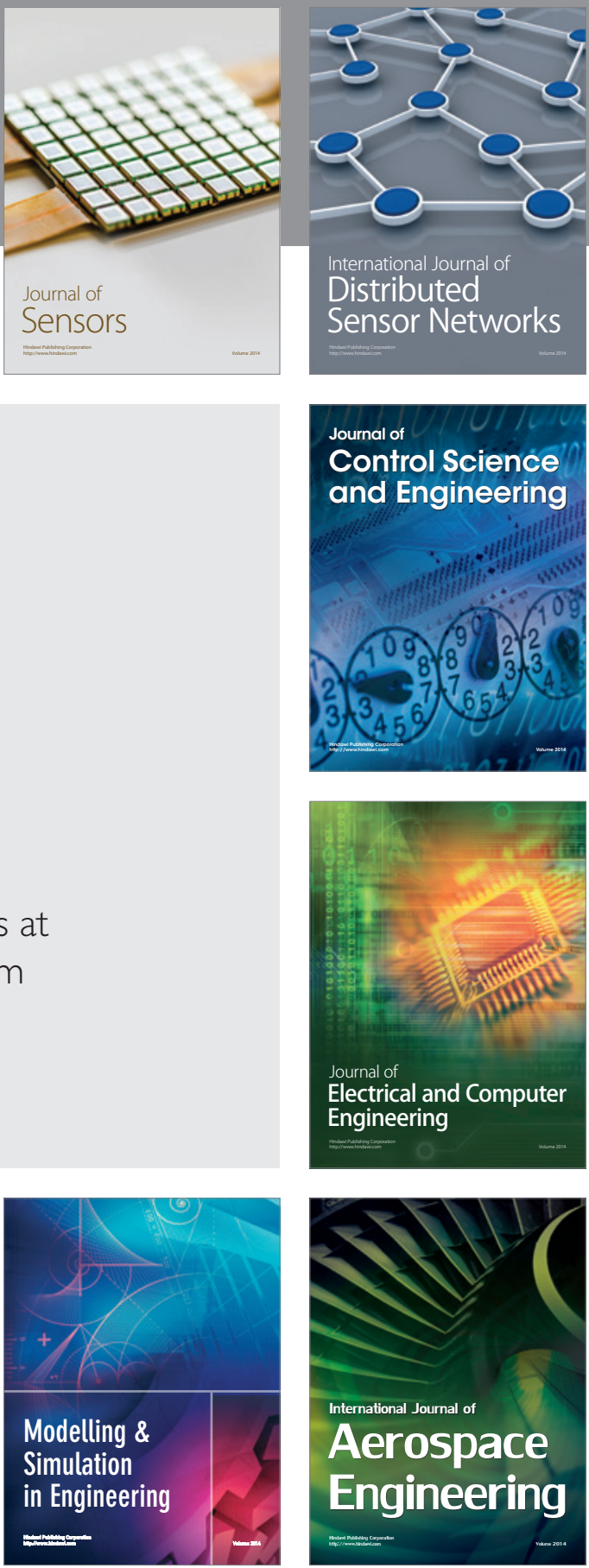

Journal of

Control Science

and Engineering
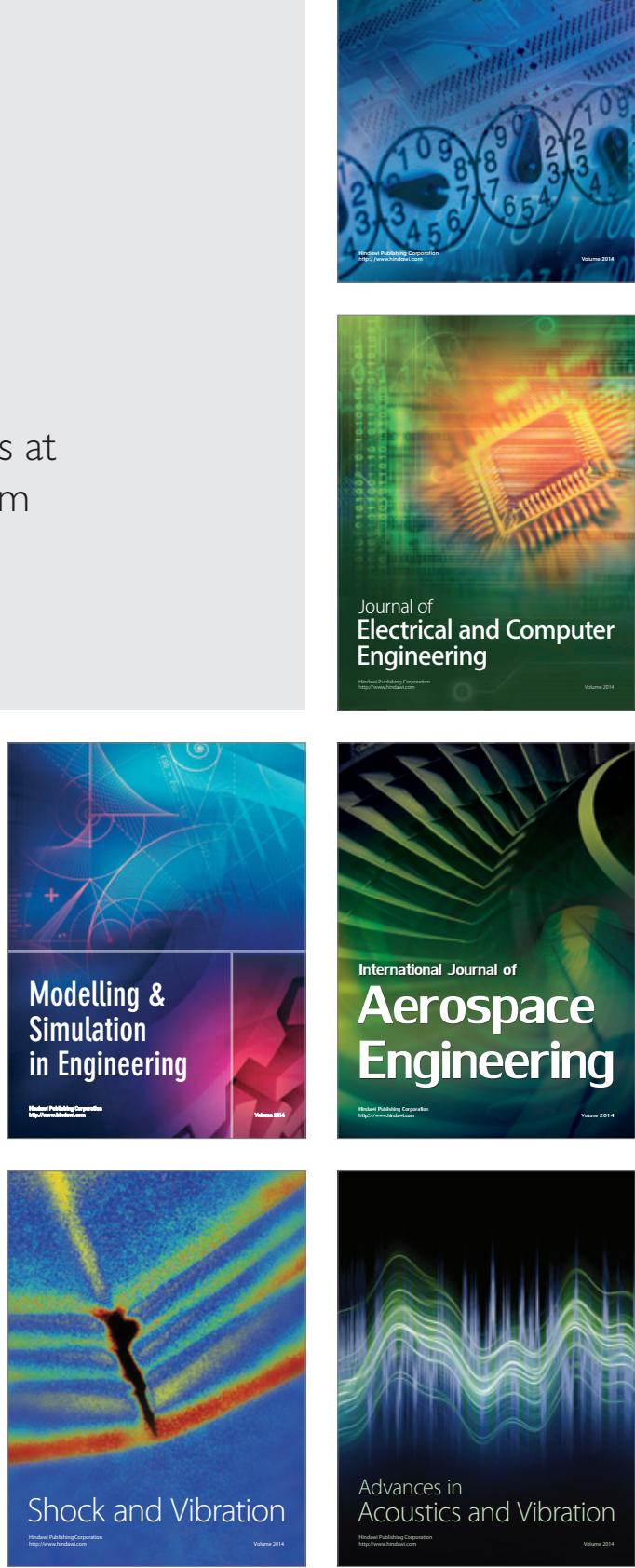